\title{
Helicobacter pylori strain-specific differences in genetic content, identified by microarray, influence host inflammatory responses
}

\author{
Dawn A. Israel, ${ }^{1}$ Nina Salama, ${ }^{2}$ Carrie N. Arnold, ${ }^{2}$ Steven F. Moss, ${ }^{3}$ Takafumi Ando, ${ }^{4}$ \\ Hans-Peter Wirth, ${ }^{4}$ Kyi T. Tham, ${ }^{5,6}$ Margorita Camorlinga, ${ }^{4}$ Martin J. Blaser, ${ }^{4,6}$ \\ Stanley Falkow, ${ }^{2}$ and Richard M. Peek, Jr. ${ }^{1,6}$ \\ ${ }^{1}$ Division of Gastroenterology, Vanderbilt University School of Medicine, Nashville, Tennessee, USA \\ ${ }^{2}$ Department of Microbiology and Immunology, Stanford University School of Medicine, Stanford, California, USA \\ ${ }^{3}$ Division of Gastroenterology, Rhode Island Hospital, Brown University School of Medicine, Providence, Rhode Island, USA \\ ${ }^{4}$ Division of Infectious Diseases, and \\ ${ }^{5}$ Department of Pathology, Vanderbilt University School of Medicine, Nashville, Tennessee, USA \\ ${ }^{6}$ Department of Veterans Affairs Medical Center, Nashville, Tennessee, USA
}

Address correspondence to: Richard M. Peek, Jr., Division of Gastroenterology, Vanderbilt University School of Medicine, C-2104 Medical Center North, Nashville, Tennessee 37232-2279, USA.

Phone: (615) 322-5200; Fax: (615) 343-6229; E-mail: richard.peek@mcmail.vanderbilt.edu.

Received for publication September 29, 2000, and accepted in revised form January 29, 2001.

\begin{abstract}
Helicobacter pylori enhances the risk for ulcer disease and gastric cancer, yet only a minority of $H$. pylori-colonized individuals develop disease. We examined the ability of two $H$. pylori isolates to induce differential host responses in vivo or in vitro, and then used an $\mathrm{H}$. pylori whole genome microarray to identify bacterial determinants related to pathogenesis. Gastric ulcer strain B128 induced more severe gastritis, proliferation, and apoptosis in gerbil mucosa than did duodenal ulcer strain G1.1, and gastric ulceration and atrophy occurred only in $\mathrm{B}^{128^{+}}$gerbils. In vitro, gerbil-passaged B128 derivatives significantly increased IL-8 secretion and apoptosis compared with G1.1 strains. DNA hybridization to the microarray identified several strain-specific differences in gene composition including a large deletion of the cag pathogenicity island in strain G1.1. Partial and complete disruption of the cag island in strain B128 attenuated induction of IL-8 in vitro and significantly decreased gastric inflammation in vivo. These results indicate that the ability of $H$. pylori to regulate epithelial cell responses related to inflammation depends on the presence of an intact cag pathogenicity island. Use of an $\mathrm{H}$. pylori whole genome microarray is an effective method to identify differences in gene content between $H$. pylori strains that induce distinct pathological outcomes in a rodent model of $H$. pylori infection.
\end{abstract}

J. Clin. Invest 107:611-620 (2001).

\section{Introduction}

Chronic gastritis induced by Helicobacter pylori enhances the risk for a diverse spectrum of diseases including duodenal and gastric ulceration and noncardia gastric cancer (1-3). However, the majority of $H$. pylori-colonized individuals remain asymptomatic; propensity to develop disease may depend on host characteristics, particular bacterial factors, or to the specific interactions between host and microbe. One histological pattern induced by $H$. pylori is pangastritis, which may subsequently progress to gastric ulceration (GU), atrophy, and intestinal metaplasia and lower the threshold for distal gastric cancer $(4,5)$. In contrast, patients with antral-predominant gastritis are more likely to develop duodenal ulcer (DU) disease, yet less likely to develop distal gastric cancer, underscoring the dichotomy of these two histological pathways (5).

Given that $H$. pylori is the most common etiologic agent of chronic gastritis, it is likely that this organism plays a causative role early in the progression to neo- plasia. Therefore, attention has focused on mechanisms by which $H$. pylori may alter cellular processes with premalignant potential, such as apoptosis and proliferation $(6,7)$. H. pylori infection of Mongolian gerbils induces gastritis $(8,9)$, which then may lead to distal gastric adenocarcinoma (10), and experimental H. pylori infection alters gastric epithelial cell apoptosis and proliferation in this model of carcinogenesis (9).

In addition to differences in host response, bacterial determinants also contribute to disease outcome. One strain-specific $H$. pylori gene is cagA, a component of the cag pathogenicity island; several genes within this island encode products that are homologs of proteins of the type IV bacterial secretion pathway $(11,12)$. In vivo, cag $A^{+}$strains augment the risk for peptic ulcer disease, atrophic gastritis, and distal gastric cancer compared with cagA- strains (13-16). A second polymorphic $H$. pylori locus is vacA, which encodes the vacuolating cytotoxin. H. pylori strains of the s1a vacA subtype are usually strongly toxigenic and $\operatorname{cag} A^{+}(17)$ and also have 
been associated with more severe clinical outcomes (17). Recently, another $H$. pylori gene, iceA, has been identified that is induced after contact with epithelial cells (18). The ice $A$ gene exists as two distinct genotypes, ice $A 1$ and ice $A 2$, and carriage of ice $A 1$ strains has been associated with peptic ulceration in some $(18,19)$, but not all (20), studies.

Although $H$. pylori cag $A^{+}$vacA s1a iceA1 strains are more frequently associated with disease, important geographic differences in susceptibility to disease exist, as clear-cut markers for $H$. pylori strains that affect Western populations have little or no predictive power in East Asian populations $(20,21)$. H. pylori isolates exhibit extensive genetic diversity involving point mutations and intragenic variation of larger segments $(22,23)$, which hinders identification of strain-specific, pathogenesis-related genes using molecular fingerprinting techniques such as RFLPs or random arbitrarily primed PCR. A more comprehensive approach to understanding the different pathogenic effects of these strains requires that each gene within the bacterial genome be interrogated simultaneously. The availability of complete genome sequences for two different $H$. pylori strains $(22,23)$ and development of microarrays containing representations of each of these genes (24) provide both of the prerequisites for this approach. Therefore, we sought to determine whether $H$. pylori isolates from patients with divergent cancer risks (i.e., GU versus DU) differentially affected epithelial cellular responses in vivo and in vitro and to identify and characterize genetic differences responsible for these events.

\section{Methods}

Animals, housing, and H. pylori challenge. Mongolian gerbils 4-8 weeks of age were purchased either from Harlan Sprague Dawley Inc. (Hsd:MON; Indianapolis, Indiana, USA) or Charles River Laboratories (Crl:[MON]BR[outbred]; Wilmington, Massachusetts, USA). All procedures were approved by the Institutional Animal Care Committee of Vanderbilt University. Gerbil-passaged cag $A^{+}$vacA s1a iceA1 strains B128 (18) and G1.1 (8) were originally isolated from patients with GU and those with DUs, respectively. Isogenic pic $B^{-}$mutants used for inoculation were generated within strain B128 as described later. Gerbils were orogastrically challenged with sterile Brucella broth or $H$. pylori and sacrificed between 1 and 52 weeks after inoculation, as described previously (8). One half of the glandular stomach was fixed in $10 \%$ neutral buffered formalin for histological examination, and the other half was homogenized in sterile PBS ( $\mathrm{pH} 7.4)$; plated on selective Trypticase soy agar plates containing vancomycin $(20 \mu \mathrm{g} / \mathrm{ml})$, nalidixic acid $(10 \mu \mathrm{g} / \mathrm{ml})$, bacitracin $(30 \mu \mathrm{g} / \mathrm{ml})$, and amphotericin B $(2 \mu \mathrm{g} / \mathrm{ml})$; and grown for 4-5 days, as described previously (8). Colonies were identified as $H$. pylori based on their characteristic morphology, and by urease and oxidase activities; colony counts were expressed as log CFU per stomach (8). B128 picB- mutant strains were also tested for kanamycin resistance, as described elsewhere (8).

Histological examination and serum antibodies to $H$. pylori. Hematoxylin and eosin sections were examined by a single experienced pathologist (KT). For semiquantitative estimates, the following parameters were graded from 0 to 3: acute and chronic inflammation, epithelial cell degeneration, and erosions (9). To quantitate parietal cell mass, total thickness of the gastric corpus glandular layer, which is composed predominantly of parietal cells, was measured in all gerbils (9). Gerbils were tail bled before sacrifice and by cardiac puncture at sacrifice, and serum Immunoglobulin G (IgG) antibody levels were measured by ELISA (8).

Immunobistochemistry. Proliferation and apoptosis were quantitated in situ using immunohistochemical stains for the proliferating cell nuclear antigen (PCNA) or terminal uridine deoxynucleotidyl nick end-labeling (TUNEL), respectively, as described (9). At least 20 well-oriented gastric glands were scored, and results are expressed as the mean number of PCNA-positive or TUNEL-positive cells per gland. Apoptosis and proliferation scores were determined on antral sections from 97 animals $(77 \mathrm{H}$. pylori-infected and 20 controls), and corpus sections from 41 animals (36 infected and five controls).

$H$. pylori strains examined in vitro. Experiments were performed with $H$. pylori strains B128 and G1.1 already described here, or with their respective gerbilpassaged derivatives. Isogenic picB/cagE (HP0544) null mutants were constructed within GU strain B128 by insertional mutagenesis, using aphA (conferring kanamycin resistance) as described previously (25). The cag island deletion mutants were generated within strain B128 by allelic exchange, using the chloramphenicol acetyl transferase (cat) gene (25). Briefly, the $5^{\prime}$ and $3^{\prime}$ flanking regions of the cag island were amplified from strain $26695 \mathrm{cag}$ (26) in which the cag island has been replaced with cat (25) using the primers 5' CCAATTTCACTCGCTATGACGGCATG 3' and $5^{\prime}$ AAGCTTTGTCTATTCTAAAATGCAAC $3^{\prime}$, and $H$. pylori B128 $\Delta$ cag mutants were generated by natural transformation and allelic exchange using this purified PCR product, as described elsewhere (26). Isogenic $p i c B$ and $\Delta c a g$ mutants were selected with either kanamycin $(25 \mu \mathrm{g} / \mathrm{ml})$ or chloramphenicol (10 $\mu \mathrm{g} / \mathrm{ml})$, respectively.

Assessment of AGS cell viability, apoptosis, and IL-8 production. AGS human gastric epithelial cells (ATCC CRL 1739) were grown in RPMI 1640 (Life Technologies Inc., Rockville, Maryland, USA) supplemented with 10\% FBS (Sigma Chemical Co., St. Louis, Missouri, USA) and $20 \mu \mathrm{g} / \mathrm{ml}$ gentamicin in an atmosphere of $5 \%$ $\mathrm{CO}_{2}$ at $37^{\circ} \mathrm{C}(25)$. For coculture experiments, $H$. pylori were grown in Brucella broth with $5 \%$ FBS for 48 hours, harvested by centrifugation $(2,000 \mathrm{~g})$, and resuspended in antibiotic-free RPMI 1640 with 10\% FBS. For quantitation of apoptosis and IL-8, H. pylori were added to cells at bacteria/cell concentrations of 100:1 and 
1,000:1, respectively, based on reports that $H$. pylori reproducibly induce apoptosis and IL-8 secretion in AGS cells at these ratios $(12,25)$. For viability, AGS cells were seeded to a density of $5 \times 10^{4}$ cells per well in 24-well plates, incubated overnight, and inoculated with $H$. pylori $\left(5 \times 10^{6} \mathrm{CFU}\right.$ per well $)$ or media alone for up to 48 hours, as described elsewhere (25). Viability was determined by trypan blue exclusion using phasecontrast microscopy. DNA fragmentation, reflecting apoptosis, was quantified using a commercially available ELISA (Roche Molecular Biochemicals, Indianapolis, Indiana, USA); AGS cells $\left(5 \times 10^{3}\right.$ per well $)$ in 96-well plates were incubated in triplicate with $H$. pylori or media alone for 24-48 hours and lysed, and after centrifugation supernatants were used for ELISA (25). For quantitation of IL-8, AGS cell monolayers in sixwell plates were cocultured with or without $H$. pylori for 24 hours in triplicate, and supernatants removed from the wells were centrifuged at $15,000 \mathrm{~g}$ before freezing at $-70^{\circ} \mathrm{C}$ as described previously (12). IL-8 protein was measured by ELISA (R\&D Systems Inc., Minneapolis, Minnesota, USA) on supernatants (12).

Microarray analysis of strains B128 and G1.1. The genomic content of GU strain B128 and DU strain G1.1 was determined using an $H$. pylori whole genome microarray containing 1,660 unique $H$. pylori genes (24). Briefly, Cy5-labeled probes were prepared for each strain from $2 \mu \mathrm{g}$ of genomic DNA using Klenow fragment, and a Cy3-labeled reference probe was prepared from a mixture of $1 \mu \mathrm{g}$ of DNA each from strains 26695 and J99, the strains used for construction of the microarray (24). $\mathrm{Cy} 5$ and $\mathrm{Cy} 3$ probes were mixed, hybridized to the microarrays, and washed (27), and arrays were scanned using an Axon scanner and further processed using Genepix 3.0 software (Axon Instruments Inc., Foster City, California, USA). Data for each channel were normalized using the Stanford Microarray Database (28). The geometric mean of the normalized red/green (R/G) ratio was computed using data from two arrays yielding one to four readings per gene (each array contains two spots per gene). Spots were excluded due to slide abnormalities or low signal ( $<100$ arbitrary units in the green channel). The cutoff for absence of a gene was a

\section{Figure 1}

Comparison of colonization density (a), serum antibody response (b), and gastric inflammation in the antrum (c) and corpus (d) in gerbils infected with $H$. pylori GU strain B128 or DU strain G1.1. (a) Animals were sacrificed 1-52 weeks after bacterial inoculation with B128 (solid symbols) or G1.1 (open symbols), and the number of CFU per stomach was determined by quantitative culture (8). Results are expressed as mean $\log _{10} \mathrm{CFU} \pm \mathrm{SD}$. (b) Anti-H. pylori IgG antibody levels in serum were determined by ELISA (8). Mean absorbance (ODU) values $\pm \mathrm{SD}$ are shown. (c) Acute $(\mathrm{Al})$ and chronic $(\mathrm{Cl})$ inflammation in the antrum of animals infected with B128 (solid symbols) or G1.1 (open symbols) was determined by histological testing, as described in the text. (d) Corpus inflammation for gerbils challenged with B128 or G1.1 was scored from 0 to 3 , and results are expressed as mean histological scores \pm SD. normalized $\mathrm{R} / \mathrm{G}$ ratio $<0.5$ based on test hybridizations (24). Data were simplified into a binary score (gene present in a given strain $=1$, absent $=0$ ) and analyzed by average hierarchical clustering using Cluster software and displayed using TreeView $(29,30)$.

Statistical analysis. The Mann-Whitney $U$ test for nonparametric data was used to compare scores between samples, whereas scores for inflammation, apoptosis, and proliferation within the same animals were compared by linear regression analysis. Apoptosis, proliferation, inflammation, and IL-8 data are presented as mean \pm SD. Significance was defined as $P \leq 0.05$.

\section{Results}

H. pylori strains induce distinct patterns of inflammation and injury in Mongolian gerbils. In total, 104 gerbils were challenged either with H. pylori DU strain G1.1 $(n=60)$, GU strain B128 $(n=24)$, or Brucella broth alone $(n=20)$. Of 84 gerbils challenged with $\mathrm{H}$. pylori, 80 (57 G1.1, 23 B128) were successfully infected, and there were no significant differences in colonization efficiency $(P=0.4)$.
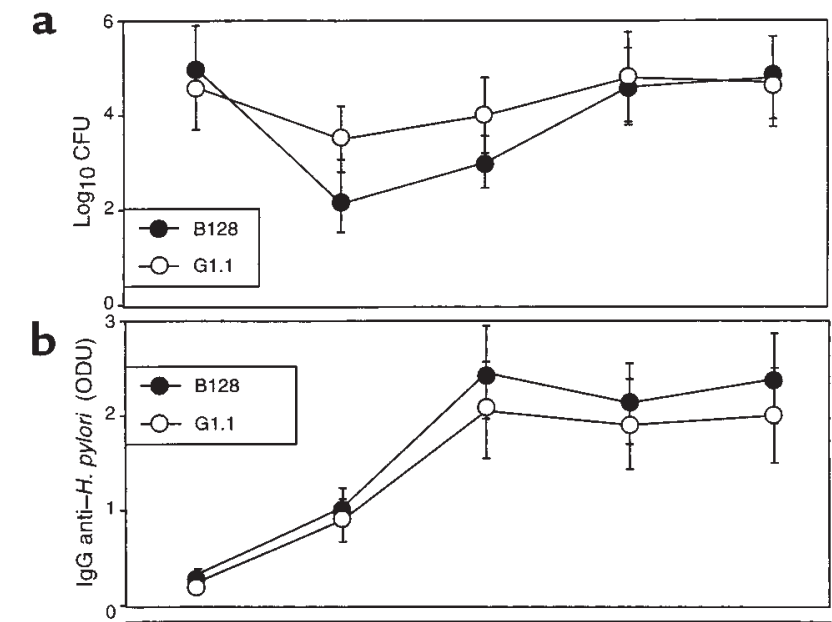

C
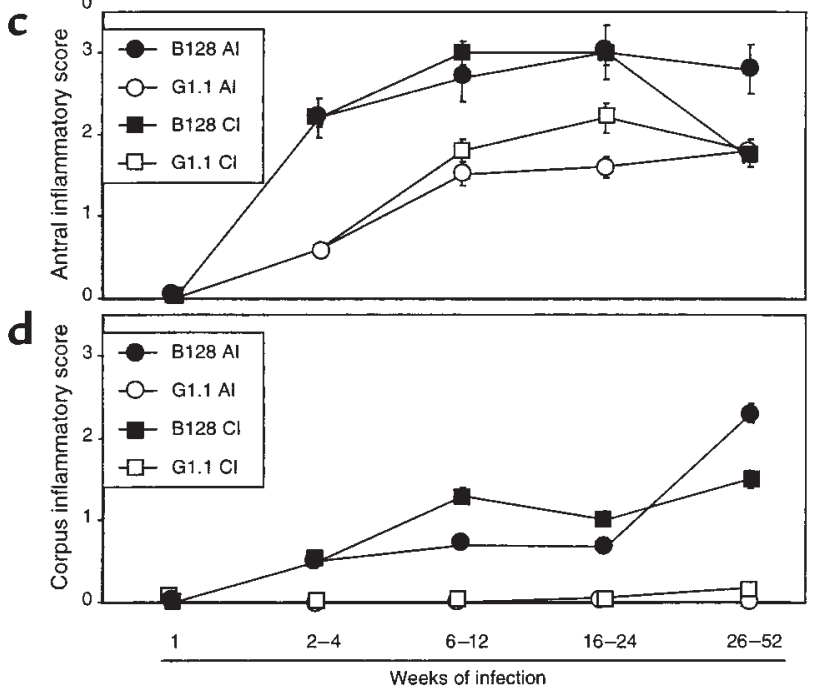
$B 128$ (GU) 3
G1.1 (DU) 1

\begin{tabular}{cccc}
5 & 6 & 3 & 6 \\
8 & 13 & 17 & 18 \\
\hline \multicolumn{3}{c}{ Number of animals }
\end{tabular}




\section{Figure 2}

H. pylori strains differentially alter gastric epithelial cell apoptosis (a) and proliferation (b) in Mongolian gerbil gastric mucosa. Apoptosis and proliferation scores at each time point are expressed as mean number of positive cells per gland in infected gerbils divided by mean scores in uninfected gerbils. Therefore, a value of 1 represents baseline. (a) Apoptosis scores were significantly higher in animals infected with GU strain B128 in the antrum (B128A; filled circles) and corpus (B128C; filled squares) 1-24 weeks after challenge compared with DU strain G1.1 (open symbols) or controls $(P=0.01$ and 0.009 , respectively); scores subsequently decreased, and by 26-52 weeks, they were not different from controls. Compared with uninfected animals, apoptosis was also significantly increased in gerbils infected with DU strain G1.1 but only in the antrum (G1.1A, open circles) and only at 2-4 weeks after inoculation $(P=0.01)$. (b) Proliferation scores increased shortly following challenge with GU strain B128 (filled symbols) and were significantly higher at all time points than either G1.1-infected (open symbols) or control animals ( $P<0.001$ for each).

We next asked whether colonization density, serum antibody response, or gastric inflammation and injury differed between the gerbil populations. There were no differences in colonization density (Figure 1a) or anti-H. pylori IgG antibody responses (Figure $1 \mathrm{~b}$ ) between B128- and G1.1-infected animals. All gerbils infected for more than 2-4 weeks developed acute and chronic antral gastritis, and scores increased until 16-24 weeks (Figure 1c). Similar to the antrum, 14 (93\%) of 15 gerbils infected with GU strain B128 for more than 2-4 weeks developed corpus gastritis, and scores steadily increased throughout the observation period (Figure 1d). In contrast, only $3(5 \%)$ of 57 gerbils infected with DU strain G1.1 had any corpus inflammation $(P \leq 0.001$ compared with B128). Acute and chronic inflammatory scores (Figure 1, $c$ and d), and the degree of epithelial cell degeneration (data not shown) were significantly $(P \leq 0.03)$ higher at all gastric sites in animals infected with B128 compared with G1.1. Thus, GU strain B128 induced a more severe and global inflammatory response than did DU strain G1.1.

Because gastric ulcer disease is associated with an increased risk for gastric adenocarcinoma (5), we next sought to determine whether GU strain B128 enhanced the development of premalignant mucosal lesions. Antral erosions and glandular displacement each were significantly $(P \leq 0.03)$ more frequent among gerbils infected with GU strain B128 (19/23 and 10/23 animals, respectively) compared with DU strain G1.1 (3/57 and $0 / 57$ animals, respectively). Gastric ulcers developed in $4(17 \%)$ of 23 and $0(0 \%)$ of 57 animals infected with GU strain B128 and DU strain G1.1, respectively $(P<0.001)$, and all ulcers were located within the gastric antrum. Given that parietal cell loss can lead to aberrant epithelial cellular differentiation (31), we determined whether the respective $H$. pylori strains variably altered parietal cell mass. Infection with GU strain B128 for more than
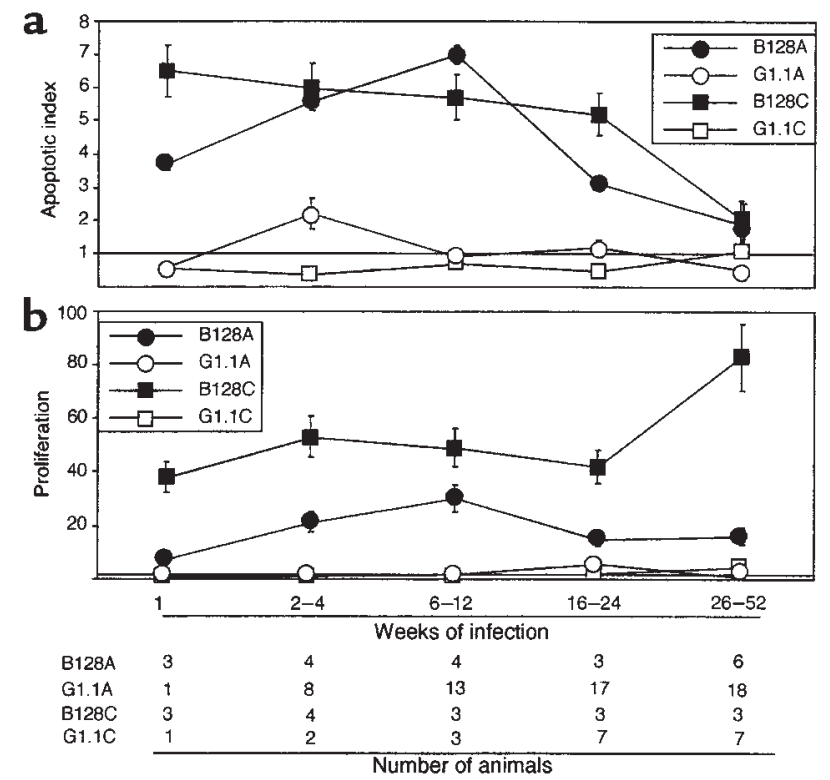

26 weeks led to parietal cell loss and glandular atrophy in $5(83 \%)$ of 6 gerbils, but these changes did not occur at any time point in the G1.1-infected animals.

H. pylori GU strain B128 induces more severe mucosal cellular imbalances. Because the severity and pattern of gastritis differed among B128- and G1.1-infected animals and because mucosal inflammation may contribute to cell turnover $(7,9)$, we next addressed whether apoptosis or proliferation was differentially affected by these $H$. pylori strains. Gerbils infected with GU strain B128 had significantly $(P \leq 0.05)$ higher antral and corpus apoptotic indices 1-24 weeks after inoculation than did broth- or G1.1-challenged animals (Figure 2a). In contrast, antral apoptosis scores in G1.1-infected animals were significantly increased only at 2-4 weeks, and corpus apoptosis scores were no different from controls (Figure 2a). We have previously shown that $H$. pylori-infected Mongolian gerbils develop hyperproliferation, although with different timing and morphological location than apoptosis (9). After challenge with GU strain B128, mucosal proliferation indices increased rapidly and were significantly higher at all time points than either G1.1-infected or control animals (Figure 2b; $P<0.001$ for each). Thus, GU strain B128 induces a more intense tissue response than G1.1 as reflected by enhanced mucosal inflammation, cell proliferation, and cell death.

H. pylori GU strain B128 enhances cytokine production and apoptosis in vitro. Given that GU strain B128 altered inflammation and cellular turnover more profoundly in vivo, we examined whether similar effects could be observed in vitro. Levels of IL-8, a proinflammatory cytokine that has potent chemoattractive and neutrophil-activating properties, are significantly related to severity of gastric inflammation among $H$. pylori-positive persons $(32,33)$. Comparing IL-8 production in AGS cells after coculture with our prototype 
H. pylori strains, GU strain B128 stimulated significantly ( $P=0.003$ versus G1.1) higher levels of IL-8 (Figure $3 \mathrm{a}$ ), results that parallel the in vivo response to this strain. AGS cells cocultured with either $H$. pylori strain had significantly $(P<0.05)$ reduced viability compared with controls (Figure 3a); however, GU strain B128 significantly $(P<0.001)$ decreased viability compared with DU strain G1.1 (Figure 3a). To determine whether the reduction in viability reflected apoptosis, we quantitated DNA fragmentation in AGS cells after bacterial coculture. H. pylori significantly increased cytoplasmic oligonucleosomal fragments compared with AGS cells alone ( $P<0.001$ for each time point; Figure 3a), and again, GU strain B128 significantly enhanced this effect $(P<0.001$; Figure 3a).

The ability of strains B128 and G1.1 to differentially alter IL-8 secretion and apoptosis in AGS cells reflected the responses to these same strains in vivo. Therefore, we next asked whether the bacterial properties responsible for these changes had been affected by prolonged in vivo growth. Similar to the results obtained with the parental strains (Figure 3a), all B128 strains after prolonged gerbil colonization induced significantly higher levels of apoptosis and IL-8 production than $\mathrm{G} 1.1$ derivatives $(P \leq 0.01$; Figure $3 \mathrm{~b}$ ), although no consistent intrafamilial strain differences were identified. These data indicate that the properties necessary for induction of epithelial cell responses remain relatively stable during persistent $H$. pylori colonization of the gastric niche.

$H$. pylori whole genome microarray reveals a partial cag island deletion in strain G1.1. Having established that strains B128 and G1.1 have different phenotypes, we next used an $H$. pylori whole genome microarray to examine differences in gene content between these prototype strains. A total of 153 strain-specific genes (98 B128-specific and 55 G1.1-specific; Figure 4) were identified in the two isolates, and $44(29 \%)$ of these (Table 1) were predicted to encode gene products with known, diverse functions such as recombination (xerD) and basic cellular metabolism (yxjE, trpA, topA3). A substantial proportion of these ORFs had significant homology to genes encoding putative restriction-modification (R-M) enzymes (Table 1), findings that substantiate recent reports (34-36) demonstrating the presence of unique complements of R-M systems within individual $H$. pylori strains. The microarray also identified major differences in gene content that localize to regions of linked genes, one of which includes a portion of the cag pathogenicity island (Figure 4). GU strain B128 contains 26 of 27 cag island genes, whereas DU strain G1.1 possesses only seven of the $27 \mathrm{cag}$ genes, indicating a strain-specific deletion of this region (Figure 4; Table 1). This dele-

\section{Figure 3}

Quantitation of IL-8 and analysis of apoptosis among preinoculation (parental) strains B128 and G1.1 (a) and their derivative progeny after gerbil passage (b). (a) AGS cells were grown alone or in the presence of H. pylori GU strain B128 (filled columns) or DU strain G1.1 (open columns). IL-8 concentrations were determined 24 hours after inoculation by ELISA. No 48-hour samples were analyzed due to the significant loss of AGS cell viability that had occurred by this time point. Cell viability and apoptosis were assessed by trypan blue exclusion and DNA fragmentation ELISA, respectively, 24 and 48 hours following inoculation. Results are expressed as the percent change in IL-8, viability, or apoptosis relative to controls. Data represent mean \pm SD of three independent experiments. ${ }^{A} P \leq 0.05$ compared with AGS cells alone. ${ }^{\mathrm{B} P} \leq 0.05$ compared with controls or with DU strain G1.1. (b) Six gerbil-passaged B128 and G1.1 isolates were selected (B128-A and G1.1-A, 2 weeks; B128-B and G1.1-B, 6-12 weeks; B128-C and G1.1-C, 16-20 weeks after challenge, respectively). Apoptosis results are expressed as levels of nucleosomal release relative to controls. IL-8 concentrations are expressed as $\mathrm{pg} / \mathrm{ml}$. Bars, SD.

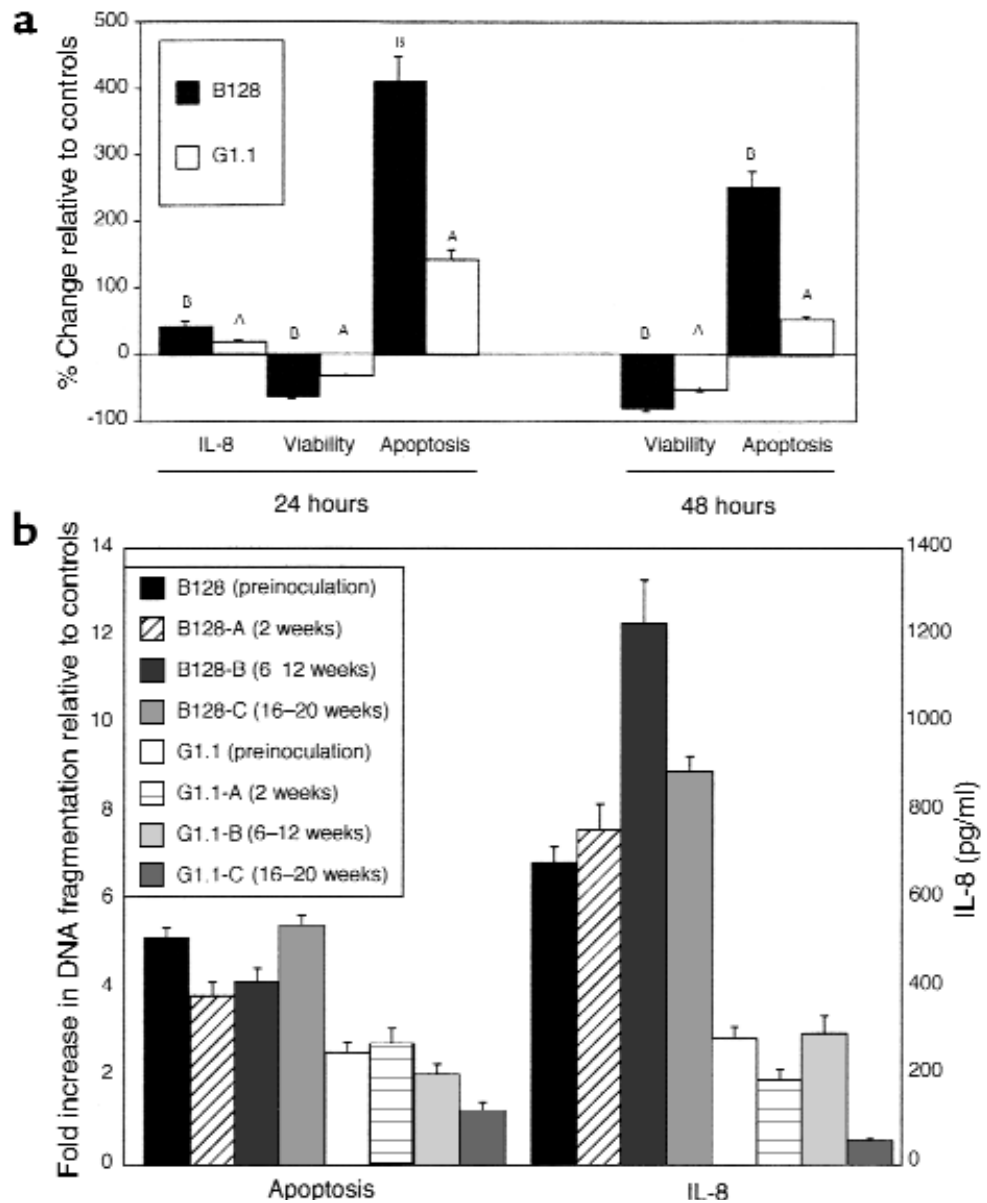




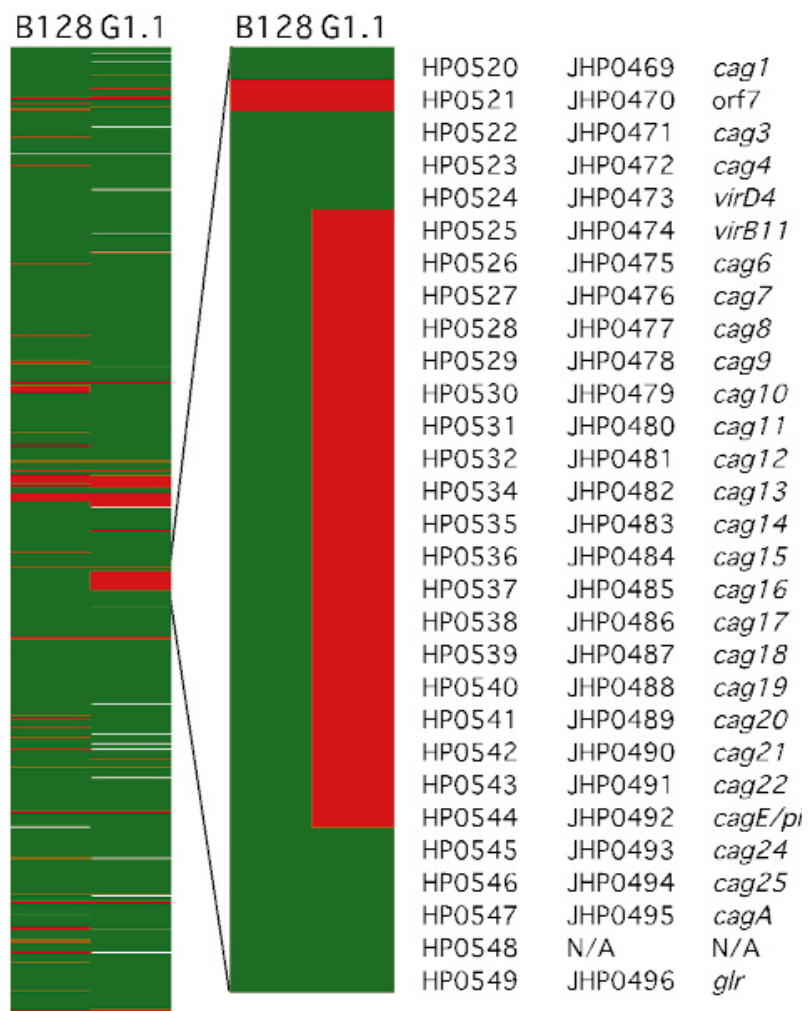

Figure 4
Comparative genomic analysis of GU strain
B128 and DU strain G1.1 using an H. pylori
whole genome microarray. The presence
(green) or absence (red) of genes was displayed
for each strain (missing data are white). The left
panel displays the entire $H$. pylori chromosome
starting at position 1 relative to the gene order
of strain 26695 (22). The right panel shows an
enhanced image of the region including the cag
pathogenicity island. The designations of genes
within the cag island are indicated. The genes
were arranged using a pseudo-gene order made
by mapping the inversions and translocations
of the J99 chromosomal sequence onto the
26695 chromosomal map.

tion consisted of contiguous genes that were located in the midregion of the island and included $\mathrm{picB} / \mathrm{cag} E$ (HP0544, JHP0492), but not cagA (HP0547, JHP0495) $(22,23)$ (Figure 4; Table 1).

Inactivation of components of the cag island attenuates $I L-8$ induction and development of gastritis. Because previous studies have demonstrated that genes within the cag island contribute to induction of IL- 8 and apoptosis in vitro $(11,12,25)$, we next disrupted $p i c B / c a g E$ and also deleted the entire cag island in GU strain B128 to determine whether these components were required for the phenotypes observed in the current study. Compared to parental strain B128, loss of picB/cagE and/or the entire cag island significantly reduced IL- 8 secretion $(P \leq 0.001$ versus B128) to levels that were similar to those induced by DU strain G1.1 (Figure 5a). Disruption of $p i c B / c a g E$ alone was sufficient to produce the phenotype obtained by deletion of the entire cag island. In contrast, the ability of these same mutant strains to induce apoptosis was not significantly different from the wild-type strain $(P=0.32$ and 0.20 versus B128, respectively; Figure 5a).

We next sought to corroborate these in vitro findings by infecting gerbils with either wild-type B128 or its isogenic pic $^{-}$derivative. Of 44 gerbils challenged with either B128 $(n=26)$ or B128 picB- $(n=18), 43$ (25 B128 and $18 \mathrm{picB}^{-}$) were successfully colonized. All strains recovered from mutant-challenged animals were kanamycin-resistant and contained the $a p h A$ cassette as determined by PCR using primers that flanked the insertion site within $p i c B$, indicating genomic stability of the antibiotic resistance cassette during in vivo colonization. There were no differences in colonization levels between the populations (data not shown). However, compared with the parental strain B128, infection with the picB- mutant significantly attenuated development of antral inflammation $(P \leq 0.03$ versus B128; Figure $5 b)$ as early as 2 weeks after challenge, and corpus inflammation by 4 weeks after infection $(P \leq 0.04$ versus B128; Figure 5c). Within the antra of $p i c B^{-}$-challenged gerbils, neutrophilic infiltration was reduced to a greater degree compared with mononuclear cell infiltration (Figure $5 \mathrm{~b}$ ). In addition to inflammation, the severity of epithelial cell degeneration and erosion was significantly ( $P \leq 0.04$ for each) decreased in gerbils infected with the picB- mutant compared with the wildtype strain (data not shown). These findings confirm our in vitro results (Figure 5a) and support the utility of whole genome microarray to identify strain-specific $H$. pylori components relevant to pathogenesis in vivo.

\section{Discussion}

Although certain $H$. pylori strains are associated with pathological outcomes, the specific mechanisms that lead to these relationships have not been fully delineated. One strain-specific $H$. pylori constituent associated with an augmented risk for ulcer disease and distal gastric cancer is the cag pathogenicity island (13-16). After H. pylori adherence to host cells, CagA is transported into the epithelial cell, where it becomes phosphorylated (37-39), and these events trigger changes in cellular morphology similar to those induced by hepatocyte growth factor (40). A CagAindependent consequence of cag island-mediated $H$. pylori-epithelial cell contact is activation of intracellular signaling pathways including $\mathrm{NF}-\kappa \mathrm{B}$, which may contribute to IL-8 secretion (41). Thus, cag $A^{+}$strains appear to be disproportionately represented among persons who develop serious sequelae of $H$. pylori carriage, and cag island genes are necessary for induction of epithelial cell responses relevant to pathogenesis.

In the current study, we examined two strains that possessed similar virulence genotypic profiles $\left(\operatorname{cag} A^{+}\right.$ 
vacA s1a iceA1), but which induced distinct phenotypic responses. GU strain B128 induced more severe inflammation within gerbil mucosa and significantly higher levels of IL-8 in vitro. Using an $H$. pylori whole genome microarray, we found that although both prototype strains possessed the cagA gene and hence were $\operatorname{cag} A^{+}$, their gene content within the $\operatorname{cag}$ island differed substantially. GU strain B128 contains a near complete copy of the island (26 of 27 genes), whereas in DU strain G1.1, a large deletion is present. These results prompted us to re-examine epithelial cell responses without the influence of cag gene products, and we confirmed the findings of previous investigators $(11,12)$ that inactivation of $p i c B / c a g E$ or deletion of the entire cag island significantly attenuated the ability of GU strain B128 to induce cytokine release in vitro. Infection of gerbils with an isogenic pic $B^{-}$deriv- ative recapitulated these events in vivo as mucosal inflammation, but not colonization, was significantly decreased in animals challenged with mutant strains compared with those infected with parental B128. The inflammatory component most profoundly affected was polymorphonuclear cell infiltration, which is consistent with the demonstration that loss of $p i c B$ is associated with decreased production of IL-8, a cytokine with potent chemotactic and activating properties for neutrophils. Our previous studies have shown that neither CagA nor VacA is required for induction of inflammation in this model of $H$. pylori-induced gastritis (9), and genomic profiling of strains B128 and G1.1 support these observations, as both strains contained $\operatorname{cag} A$ and $v a c A$. These findings underscore and confirm the importance of the entire cag island as a bacterial locus related to high-grade host responses

Table 1

Strain-specific B128 and G1.1 genes with putative functions

\begin{tabular}{|c|c|c|c|c|c|}
\hline \multicolumn{2}{|c|}{ Strain $^{A}$} & \multirow[b]{2}{*}{ Gene designation $^{B}$} & \multirow[b]{2}{*}{ Gene description ${ }^{C}$} & \multicolumn{2}{|c|}{ Present $t^{\mathrm{D}}$} \\
\hline 26695 & J99 & & & B128 & G1. \\
\hline & JHP0045 & & Putative type II DNA modification enzyme & + & - \\
\hline & JHP0046 & & Putative type II restriction enzyme & + & - \\
\hline & JHP0585 & & Putative 3-hydroxyacid dehydrogenase & + & - \\
\hline & JHP0931 & topA3 & Topoisomerase I & + & - \\
\hline & JHP1422 & hsdS3a & Putative type I restriction enzyme (specificity subunit) & + & - \\
\hline HP0459 & & virB4 & virB4 homolog & + & - \\
\hline HP0483 & JHP0435 & & Type II DNA modification enzyme & + & - \\
\hline HP0525 & JHP0474 & virB11 & virB11 homolog & + & - \\
\hline HP0526 & JHP0475 & cag6 & cag pathogenicity island protein & + & - \\
\hline HP0527 & JHP0476 & cag7 7 & cag pathogenicity island protein & + & - \\
\hline HP0528 & JHP0477 & cag8 & cag pathogenicity island protein & + & - \\
\hline HP0529 & JHP0478 & cag9 & cag pathogenicity island protein & + & - \\
\hline HP0530 & JHP0479 & cag10 & cag pathogenicity island protein & + & - \\
\hline HP0531 & JHP0480 & cag11 & cag pathogenicity island protein & + & - \\
\hline HP0532 & JHP0481 & cag12 & cag pathogenicity island protein & + & - \\
\hline HP0534 & JHP0482 & cag13 & cag pathogenicity island protein & + & - \\
\hline HP0535 & JHP0483 & cag14 & cag pathogenicity island protein & + & - \\
\hline HP0536 & JHP0484 & cag15 & cag pathogenicity island protein & + & - \\
\hline HP0537 & JHP0485 & cag16 & cag pathogenicity island protein & + & - \\
\hline HP0538 & JHP0486 & cag17 & cag pathogenicity island protein & + & - \\
\hline HP0539 & JHP0487 & cag18 & cag pathogenicity island protein & + & - \\
\hline HP0540 & JHP0488 & cag19 & cag pathogenicity island protein & + & - \\
\hline HP0541 & JHP0489 & $\operatorname{cag} 20$ & cag pathogenicity island protein & + & - \\
\hline HP0542 & JHP0490 & cag21 & cag pathogenicity island protein & + & - \\
\hline HP0543 & JHP0491 & cag22 & cag pathogenicity island protein & + & - \\
\hline HP0544 & JHP0492 & cag23/cagE/picB & cag pathogenicity island protein & + & - \\
\hline HP0962 & & $\operatorname{acp} P$ & acyl carrier protein & + & - \\
\hline HP0995 & JHP0941 & xerD & integrase/recombinase & + & - \\
\hline HP1000 & & & PARA protein & + & - \\
\hline HP1193 & & & aldo-keto reductase & + & - \\
\hline HP1370 & & $\bmod$ & type III restriction enzyme M protein & + & - \\
\hline HP1371 & JHP1285 & & type III restriction enzyme $R$ protein & + & - \\
\hline HP1517 & & Eco57IR & type lis restriction enzyme $\mathrm{R}$ and $\mathrm{M}$ protein & + & - \\
\hline & JHP0164 & & putative restriction enzyme & - & + \\
\hline HP0091 & JHP0084 & $h s d R$ & type II restriction enzyme R protein & - & + \\
\hline HP0289 & JHP0274 & & toxin-like outer membrane protein & - & + \\
\hline HP0315 & & vapD & virulence associated protein $\mathrm{D}$ & - & + \\
\hline HP0317 & & omp9 & outer membrane protein & - & + \\
\hline HP0428 & & & phage/colicin/tellurite resistance cluster & - & + \\
\hline HP0692 & JHP0636 & $y \times j E / s c o B$ & 3-oxoadipate coA-transferase subunit B & - & + \\
\hline HP0896 & JHP1164 & $b a b B$ & outer membrane protein & - & + \\
\hline HP1009 & & & site-specific recombinase & - & + \\
\hline HP1277 & JHP1198 & $\operatorname{trp} A$ & tryptophan synthase & - & + \\
\hline HP1404 & & hsdS & type I restriction enzyme S protein & - & + \\
\hline
\end{tabular}

A Unique identifier from $H$. pylori genes in annotated sequences of 26695 and J99. BPutative gene name if available. ${ }^{C}$ Description of putative gene function. DPresence $(+)$ as determined by an average fluorescence ratio of greater or equal to 0.5 from two independent hybridizations. Absence (-) was defined as an average fluorescence ratio of less than 0.5 . 

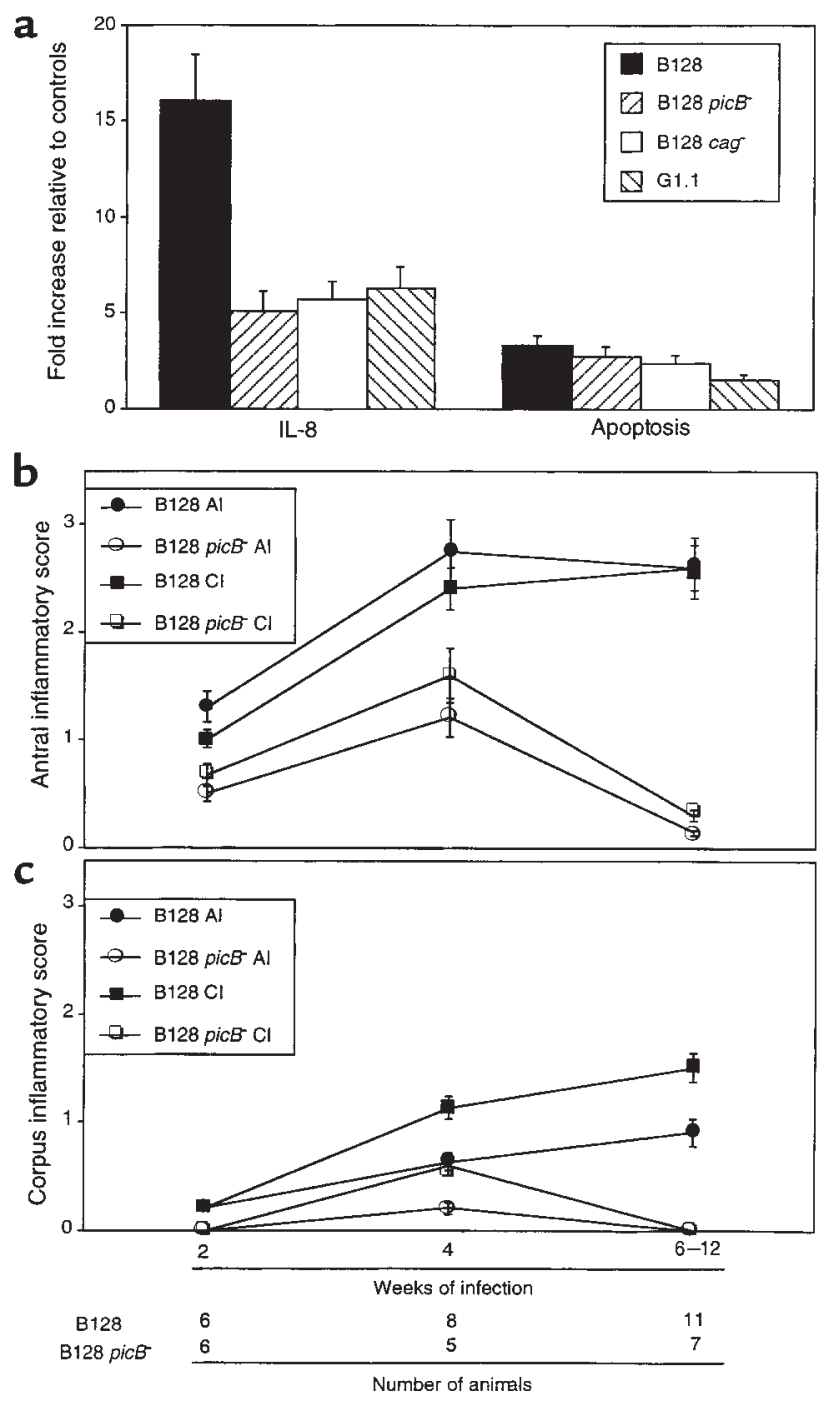

Figure 5

Inactivation of cag pathogenicity island components attenuates induction of IL-8 (a) and gastric inflammation (b and $\mathbf{c})$. (a) Quantitation of IL-8 production and apoptosis in AGS cells incubated with parental $\mathrm{GU}$ strain B128, an isogenic picB- (cagE-) mutant of B128, B128 $\mathrm{cag}^{-}$, which lacks the entire cag island, and DU strain G1.1. IL-8 concentrations and apoptosis were determined 24 hours after $\mathrm{H}$. pylori inoculation and are expressed as levels of induction relative to controls. Bars, $\mathrm{SD}$. (b) Acute $(\mathrm{Al})$ and chronic $(\mathrm{Cl})$ inflammation in the antrum of animals after 2-12 weeks of infection with B128 (filled symbols) or B128 pic $B^{-}$(open symbols) was determined by histological testing, as described in the text. (c) Corpus inflammation for gerbils challenged with $\mathrm{B} 128$ or $\mathrm{B} 128$ pic $^{-}$was scored from 0 to 3 by a single pathologist, and results are expressed as mean histological scores \pm SD.

and provide further evidence that strain-specific bacterial components identified by microarray are relevant to pathology within the gastric niche.

Many of the genetic differences identified between the two studied strains involved the cag island; however, deletion of this locus did not significantly affect the ability of GU strain B128 to induce apoptosis in vitro. Other $H$. pylori components that may induce apoptosis include urease, a highly conserved $H$. pylori constituent, which can induce apoptosis in vitro by binding to MHC class II molecules expressed on the surfaces of gastric epithelial cells (42). However, although $H$. pylori can directly induce apoptosis in isolated in vitro systems, apoptosis in vivo is likely to be regulated by host mediators present within inflamed mucosa. The immune response elicited by $\mathrm{H}$. pylori in humans and by Helicobacter felis in mice is Th1-predominant $(43,44)$, and IFN- $\gamma$, a Th1 lymphocyte-derived cytokine that is increased within colonized mucosa, is synergistic with H. pylori in inducing Fas-Fas ligand-regulated (FasLregulated) apoptosis of gastric epithelial cells in vitro $(45,46) . H$. pylori infection of IFN- $\gamma$-deficient mice leads to decreased levels of gastric inflammation compared with wild-type mice $(47,48)$, and in vivo neutralization of IFN- $\gamma$ in mice infected with $H$. felis similarly reduces the severity of gastritis (43). Adoptive transfer of Th1 cells from $H$. pylori-infected mice into infected recipients increases the severity of gastritis (49), and concurrent helminth infection modulates a proinflammatory Th1-mediated murine gastritis and atrophy response to $H$. felis toward a less injurious Th2-type response (50). H. felis infection of Fas-deficient mice is also associated with reduced levels of inflammation compared with infected wild-type mice, and mucosal apoptosis scores are decreased in parallel (51).

One hypothesis suggested by the previous findings described here is that Th1-derived cytokines (i.e., IFN- $\gamma$ ) within inflamed mucosa may increase apoptosis by regulating Fas-FasL interactions between inflammatory cells and gastric epithelial cells. However, additional host genetic factors also may contribute to the ability of $H$. pylori to alter apoptosis in vivo. Mice lacking secretory phospholipase $\mathrm{A}_{2}$ that are infected by $H$. felis develop increased epithelial cell apoptosis (52). Apoptosis in response to H. pylori also may reflect heterogeneity of class II MHC host genotypes, as binding of $\mathrm{H}$. pylori to these IFN- $\gamma$-inducible molecules can directly stimulate apoptosis (42). ElOmar et al. have recently identified particular polymorphisms of the human IL- $1 \beta$ gene promoter that are genetic risk factors for both precursor lesions of gastric cancer (atrophic gastritis) as well as gastric adenocarcinoma per se among $H$. pylori-infected persons (53). IL- $1 \beta$ can stimulate multiple intracellular signaling pathways involved in apoptosis, including Fas and NF- $\mathrm{\kappa B}(41,45,46)$, and thus differing levels of IL-1 $\beta$ expression within $H$. pylori-colonized gastric mucosa may regulate levels of apoptosis in vivo.

IL-1 $\beta$ is also a potent inhibitor of gastric acid secretion, and polymorphisms within its promoter region may differentially influence additional physiological events that dictate disease outcomes among $H$. pylori-colonized populations. Two recent investigations have revealed that acid outputs in gerbils chronically infected with $H$. pylori are either no different (54) or reduced (55) compared with uninfected controls. Further, mucosal IL-1 $\beta$ mRNA levels reciprocally increase as acidity decreases and administration of recombinant 
IL-1 receptor antagonist normalizes acid outputs among gerbils carrying $H$. pylori (55). Collectively, these data indicate that the host inflammatory response is an important determinant of specific pathological outcomes among $H$. pylori-infected populations.

Understanding H. pylori diversification and adaptation during long-term colonization is crucial toward delineating mechanisms by which these organisms may lead to disease. As evidenced by our results, the use of microarray-based comparative genomics provides a powerful tool for identifying strain-specific factors that may contribute to phenotypic variability among $H$. pylori isolates assumed to have similar genetic composition. One limitation of this technique, however, is that it cannot detect small deletions, point mutations, deletions within intergenic regions, rearrangements, ORFs that are not present in either strain 26695 or J99, or deletions of homologous repetitive elements. However, as microarrays of greater resolution are developed and new strain-specific ORFs are identified, it should soon be possible to determine the relative frequency and importance of these additional sources of genetic variability. Genotypic markers could then be developed to not only to identify individuals at risk for specific clinical sequelae of infection, but also to permit selective targeting of therapy for disease prevention.

In conclusion, we have shown that $H$. pylori strains with similar virulence markers can induce distinct patterns of gastric inflammation and injury in an animal model of $H$. pylori-induced carcinogenesis and that these findings can be corroborated in vitro and in vivo. DNA hybridization to an $H$. pylori whole genome microarray revealed that differences in the ability of $H$. pylori strains to induce epithelial cell responses related to inflammation are dependent on the presence of an intact cag pathogenicity island, although the specific function of numerous additional strain-specific loci and their significance in pathogenesis remain to be determined.

\section{Acknowledgments}

Supported in part by the NIH (KO8 DK02381, R29 CA77955, R01 DK50837, and RO1 AI38459), and by the Medical Research Service of the Department of Veterans Affairs. We thank Tyler Richmond and Uma Krishna for excellent technical assistance.

1. Peterson, W.L. 1991. Helicobacter pylori and peptic ulcer disease. N. Engl. J. Med. 324:1043-1048.

2. Nomura, A., et al. 1991. Helicobacter pylori infection and gastric carcinoma among Japanese Americans in Hawaii. N. Engl. J. Med. 325:1132-1136.

3. Parsonnet, J., et al. 1991. Helicobacter pylori infection and the risk of gastric carcinoma. N. Engl. J. Med. 325:1127-1131.

4. Correa, P., et al. 1990. Gastric precancerous process in a high risk population: cohort follow-up. Cancer Res. 50:4737-4740.

5. Hansson, L.E., et al. 1996. The risk of stomach cancer in patients with gastric or duodenal ulcer disease. N. Engl. J. Med. 335:242-249.

6. Moss, S.F., Calam, J., Agarwal, B., Wang, S., and Holt, P.R. 1996. Induction of gastric epithelial apoptosis by Helicobacter pylori. Gut. 38:498-501.

7. Peek, R.M., Jr., et al. 1997. Helicobacter pylori cagA+ strains and dissoci- ation of gastric epithelial cell proliferation from apoptosis. J. Natl. Cancer Inst. 89:863-868.

8. Wirth, H.P., Beins, M.H., Yang, M., Tham, K.T., and Blaser, M.J. 1998. Experimental infection of Mongolian gerbils with wild-type and mutant Helicobacter pylori strains. Infect. Immun. 66:4856-4866.

9. Peek, R.M., et al. 2000. Helicobacter pylori alters gastric epithelial cell cycle events and gastrin secretion in Mongolian gerbils. Gastroenterology. 118:48-59.

10. Watanabe, T., Tada, M., Nagai, H., Sasaki, S., and Nakao, M. 1998. Helicobacter pylori infection induces gastric cancer in Mongolian gerbils. Gastroenterology. 115:642-648.

11. Censini, S., et al. 1996. cag, a pathogenicity island of Helicobacter pylori, encodes type I-specific and disease-associated virulence factors. Proc. Natl. Acad. Sci. USA. 93:14648-14653.

12. Tummuru, M.K., Sharma, S.A., and Blaser, M.J. 1995. Helicobacter pylori picB, a homologue of the Bordetella pertussis toxin secretion protein, is required for induction of IL-8 in gastric epithelial cells. Mol. Microbiol. 18:867-876.

13. Crabtree, J.E., et al. 1991. Mucosal IgA recognition of Helicobacter pylori $120 \mathrm{kDa}$ protein, peptic ulceration, and gastric pathology. Lancet. 338:332-335.

14. Kuipers, E.J., Perez-Perez, G.I., Meuwissen, S.G., and Blaser, M.J. 1995. Helicobacter pylori and atrophic gastritis: importance of the cagA status. J. Natl. Cancer Inst. 87:1777-1780.

15. Crabtree, J.E., et al. 1993. Systemic and mucosal humoral responses to Helicobacter pylori in gastric cancer. Gut. 34:1339-1343.

16. Blaser, M.J., et al. 1995. Infection with Helicobacter pylori strains possessing $\operatorname{cag} A$ is associated with an increased risk of developing adenocarcinoma of the stomach. Cancer Res. 55:2111-2115.

17. Atherton, J.C., et al. 1995. Mosaicism in vacuolating cytotoxin alleles of Helicobacter pylori: Association of specific vacA types with cytotoxin production and peptic ulceration. J. Biol. Chem. 270:17771-17777.

18. Peek, R.M., Jr., et al. 1998. Adherence to gastric epithelial cells induces expression of a Helicobacter pylori gene, iceA, that is associated with clinical outcome. Proc. Assoc. Am. Physicians. 110:531-544.

19. van Doorn, L.J., et al. 1998. Clinical relevance of the cagA, vacA, and ice $A$ status of Helicobacter pylori. Gastroenterology. 115:58-66.

20. Ito, Y., et al. 2000. Sequence analysis and clinical significance of the iceA gene from Helicobacter pylori strains in Japan. J. Clin. Microbiol. 38:483-488.

21. Maeda, S., et al. 1997. High seropositivity of anti-CagA antibody in Helicobacter pylori-infected patients irrelevant to peptic ulcers and normal mucosa in Japan. Dig. Dis. Sci. 42:1841-1847.

22. Tomb, J.F., et al. 1997. The complete genome sequence of the gastric pathogen Helicobacter pylori. Nature. 388:539-547.

23. Alm, R.A., et al. 1999. Genomic-sequence comparison of two unrelated isolates of the human gastric pathogen Helicobacter pylori. Nature. 397:176-180.

24. Salama, N., et al. 2000. A whole genome microarray reveals genetic diversity among Helicobacter pylori strains. Proc. Natl. Acad. Sci. USA. 97:14668-14673.

25. Peek, R.M., Jr., et al. 1999. Helicobacter pylori strain-specific genotypes and modulation of the gastric epithelial cell cycle. Cancer Res. 59:6124-6131.

26. Akopyants, N.S., et al. 1998. Analyses of the cag pathogenicity island of Helicobacter pylori. Mol. Microbiol. 28:37-53.

27. Eisen, M.B., and Brown, P.O. 1999. DNA arrays for analysis of gene expression. Methods Enzymol. 303:179-205.

28. Stanford Microarray Database. http://genome-www4.stanford.edu/ MicroArray/help/results normalization.html.

29. http://www.microarrays.org/software.html. Accessed August, 2000.

30. Eisen, M.B., Spellman, P.T., Brown, P.O., and Botstein, D. 1998. Cluster analysis and display of genome-wide expression patterns. Proc. Natl. Acad. Sci. USA. 95:14863-14868.

31. Guruge, J.L., et al. 1998. Epithelial attachment alters the outcome of Helicobacter pylori infection. Proc. Natl. Acad. Sci. USA. 95:3925-3930.

32. Peek, R.M., Jr., et al. 1995. Heightened inflammatory response and cytokine expression in vivo to cagA+Helicobacter pylori strains. Lab. Invest. 73:760-770.

33. Crabtree, J.E., Peichl, P., Wyatt, J.I., Stachl, U., and Lindley, I.J. 1993. Gastric interleukin-8 and IgA IL-8 autoantibodies in Helicobacter pylori infection. Scand. J. Immunol. 37:65-70.

34. Akopyants, N.S., et al. 1998. PCR-based subtractive hybridization and differences in gene content among strains of Helicobacter pylori. Proc. Natl. Acad. Sci. USA. 95:13108-13113.

35. Xu, Q., Morgan, R.D., Roberts, R.J., and Blaser, M.J. 2000. Identification of type II restriction and modification systems in Helicobacter pylori reveals their substantial diversity among strains. Proc. Natl. Acad. Sci. USA. 97:9671-9676.

36. Ando, T., et al. 2000. Restriction-modification system differences in Helicobacter pylori are a barrier to interstrain plasmid transfer. Mol. Microbiol. 37:1052-1065. 
37. Odenbreit, S., et al. 2000. Translocation of Helicobacter pylori CagA into gastric epithelial cells by type IV secretion. Science. 287:1497-1500.

38. Stein, M., Rappuoli, R., and Covacci, A. 2000. Tyrosine phosphorylation of the Helicobacter pylori CagA antigen after cag-driven host cell translocation. Proc. Natl. Acad. Sci. USA. 97:1263-1268.

39. Asahi, M., et al. 2000. Helicobacter pylori CagA protein can be tyrosine phosphorylated in gastric epithelial cells. J. Exp. Med. 191:593-602.

40. Segal, E.D., Cha, J., Lo, J., Falkow, S., and Tompkins, L. 1999. Altered states: involvement of phosphorylated CagA in the induction of host cellular growth changes by Helicobacter pylori. Proc. Natl. Acad. Sci. USA. 96:14559-14564

41. Keates, S., Hitti, Y.S., Upton, M., and Kelly, C.P. 1997. Helicobacter pylori infection activates NF-kappa B in gastric epithelial cells. Gastroenterology. 113:1099-1109.

42. Fan, X., et al. 2000. Helicobacter pylori urease binds to class II MHC on gastric epithelial cells and induces their apoptosis. J. Immunol. 165:1918-1924

43. Mohammadi, M., Czinn, S., Redline, R., and Nedrud, J. 1996. Helicobacter-specific cell-mediated immune responses display a predominant $\mathrm{Th} 1$ phenotype and promote a delayed-type hypersensitive response in the stomachs of mice. J. Immunol. 156:4729-4738.

44. Bamford, K.B., et al. 1998. Lymphocytes in the human gastric mucosa during Helicobacter pylori have a T helper cell 1 phenotype. Gastroenterology. 114:482-492.

45. Wagner, S., et al. 1997. Regulation of gastric epithelial cell growth by Helicobacter pylori: evidence for a major role of apoptosis. Gastroenterology. 113:1836-1847.

46. Wang, J., et al. 2000. Helicobacter pylori modulates lymphoepithelial cell interactions leading to epithelial cell damage through Fas/Fas ligand interactions. Infect. Immun. 68:4303-4311.

47. Sawai, N., et al. 1999. Role of gamma interferon in Helicobacter pyloriinduced gastric inflammatory responses in a mouse model. Infect. Immun. 67:279-285.

48. Smythies, L.E., et al. 2000. Helicobacter pylori-induced mucosal inflammation is Th1 mediated and exacerbated in IL-4, but not IFN- $\gamma$, gene deficient mice. J. Immunol. 165:1022-1029.

49. Mohammadi, M., Nedrud, J., Redline, R., Lycke, N., and Czinn, S.J. 1997. Murine CD4 T-cell responses to Helicobacter infection: TH1 cells enhance gastritis and $\mathrm{TH} 2$ cells reduce bacterial load. Gastroenterology. 113:1848-1857.

50. Fox, J.G., et al. 2000. Concurrent enteric helminth infection modulates inflammation and gastric immune responses and reduces Helicobacterinduced gastric atrophy. Nat. Med. 6:536-542.

51. Houghton, J., Bloch, L.M., Goldstein, M., von Hagen, S., and Korah, R.M. 2000. In vivo disruption of the Fas pathway abrogates gastric growth alterations secondary to Helicobacter infection. J. Infect. Dis. 182:856-864.

52. Wang, T.C., et al. 1998. Mice lacking secretory phospholipase A2 show altered apoptosis and differentiation with Helicobacter felis infection. Gastroenterology. 114:675-689.

53. El-Omar, E.M., et al. 2000. Interleukin-1 polymorphisms associated with increased risk of gastric cancer. Nature. 404:398-402.

54. Keto, Y., Takahashi, S., and Okabe, S. 1999. Healing of Helicobacter pyloriinduced gastric ulcers in Mongolian gerbils: combined treatment with omeprazole and clarithromycin. Dig. Dis. Sci. 44:257-265.

55. Takashima, M., Furuta, T., Hanai, H., Sugimura, H., and Kaneko, E. 2000. The effects of Helicobacter pylori on gastric acid secretion and serum gastrin levels in Mongolian gerbils with reference to interleukin-1 $\beta$. Gastroenterology. 118:A746. (Abstr.) 